\title{
Reimagining third phase formation as the miscibility gap of a molecular solution
}

\author{
Michael J. Servis, ${ }^{*, \dagger}$ David T. Wu, Jenifer C. Shafer,,+ and Aurora E. Clark ${ }^{*, \dagger}$, \\ $\dagger$ Department of Chemistry, Washington State University, Pullman, WA \\ $\ddagger$ Department of Chemistry, Colorado School of Mines, Golden, CO \\ IDepartment of Chemical and Biological Engineering, Colorado School of Mines, Golden, \\ $\mathrm{CO}$ \\ $\S$ Pacific Northwest National Laboratory, Richland, WA \\ E-mail: michael.servis@wsu.edu; auclark@wsu.edu
}

\begin{abstract}
Liquid/liquid phase transitions are inherent to multicomponent solutions, which often contain a diversity of intermolecular interactions between their molecular constituents. In one such example, a phase transition is observed in liquid/liquid extraction where the nonpolar organic phase separates into two phases under sufficiently high metal and acid extraction by the amphiphilic extractant molecule. This deleterious phenomenon, known as third phase formation, complicates processing and limits efficiency. While empirically well documented, the molecular origin of this phenomenon is not understood. The prevailing conceptualization of the organic phase treats it as a microemulsion where extractant molecules form reverse micelles that contain the extracted aqueous solutes in their polar cores. Yet recent studies indicate that a microemulsion paradigm is insufficient to describe molecular aggregation in some solvent extraction systems, implying that an alternative description of aggregation, and explanation for third phase formation, is needed. In this study, we demonstrate that the
\end{abstract}


formation of a third phase is consistent with crossing the liquid-liquid miscibility gap for a molecular solution rather than a Winsor II to Winsor III transition as presumed in the microemulsion paradigm. This insight is provided by using a graph theoretic methodology, generalizable to other complex multicomponent molecular solutions, to identify the onset of phase splitting. This approach uses connectivity obtained from molecular dynamics simulation to correlate the molecular-scale association of extractants and extracted solutes to the solution phase behavior using percolation theory. The method is applied to investigate a solvent extraction system relevant to ore purification and used nuclear fuel recycling: tri-n-butyl phosphate/uranyl nitrate/water/nitric acid/ndodecane. In analogy to a molecular solution, immediately preceding the liquid-liquid coexistence curve from the single phase region, the metal-ligand complexes percolate. This demonstrates that describing this solution with microemulsion chemistry is neither applicable nor broadly required to explain third phase formation. Additionally, the method developed herein can predict third phase formation phase boundaries from simulation for this and potentially other solvent extraction systems.

\section{Introduction}

Complex multicomponent solutions can exhibit liquid-liquid phase transitions whose characterization requires mapping the phase boundaries as a function of different system variables. Examples can be drawn from the formation of biological membranes ${ }^{12}$ to separations science. ${ }^{[3}$ Fundamentally, a number of significant challenges exist toward understanding and characterizing these phenomena, either through identifying the most appropriate conceptual model that describes the phase behavior, or elucidating the influence of molecular-scale speciation and organization upon the macroscopic phase transition. A significant need exists for generalized methods that can provide such insights.

A quintessential example found within separations science is liquid/liquid extraction (LLE), which is the predominant industrial technique for the targeted recovery of met- 
als. ${ }^{[4}$ Liquid/liquid extraction commonly utilizes a neutral solvating extractant molecule in a nonpolar organic solvent to selectively extract metal cations with charge neutralizing anions from an acidic aqueous media. Metal-ligand complex aggregation affects their organic phase solubility and impacts the limiting organic concentration (LOC) of the metal before the onset of phase instability. ${ }^{[5}$ In so-called "third phase formation," the organic phase splits into two phases: a solute-rich "heavy" organic phase and a solute-poor "light" organic phase. This undesirable phase transition is a processing impediment that limits the efficiency of solvent extraction systems. Much work on understanding third phase formation has focused upon LLE applications that pertain to the nuclear fuel cycle. Here, the industry standard Plutonium and Uranium Reduction EXtraction (PUREX) liquid/liquid extraction process uses the ubiquitous amphiphilic extractant molecule tri-n-butyl phosphate (TBP) to selectively extract uranium $\mathrm{U}(\mathrm{VI})$ from a nitric acid aqueous media into an aliphatic solvent in the form $(\mathrm{TBP})_{2}\left(\mathrm{UO}_{2}\right)\left(\mathrm{NO}_{3}\right)_{2} \cdot \underline{6}$

Interpreting organic phase aggregation from experimental or simulation data mandates a choice of modeling paradigm between a molecular solution or a microemulsion, which we compare and contrast in Figure 1. Aggregation in the organic phase has been typically conceptualized as a microemulsion consisting of reverse micelles composed of extractant molecules solvating a polar core containing the extracted aqueous solutes. $\frac{37-10}{10}$ Small angle neutron (SANS) and X-ray (SAXS) scattering have been used to study the aggregation of extracted TBP/uranyl nitrate adducts in organic solvents.11 11 In those studies, extracted metal-ligand complexes, or stoichiometric solvates, are treated as reverse micelles. Under this description of the organic phase, third phase formation is analogous to a transition from a two phase water-in-oil micellar system (Winsor II) to a three phase system (Winsor III) with the bicontinuous phase corresponding to the heavy organic phase. $\frac{310}{10}$ The reverse micelles of water-in-oil microemulsions have been modeled as colloidal particles, where micelle-micelle interactions are described by a necessarily simplistic potential energy function, $\mathrm{U}(\mathrm{r})$, such as that between hard spheres or ellipsoids with surface attraction. Those models are typically 
Figure 1: For association of metal-ligand complexes (this study), or the formation of hydrogen bonded clusters, $\frac{16] 17}{16}$ two modeling paradigms for organic phase solutions are contrasted: microemulsions (left) and molecular solutions (right). Differences in model length scales are illustrated in the top row. The middle row highlights properties which inform modeling for microemulsions (reverse micelle $\mathrm{U}(\mathrm{r})$, critical aggregation concentration, interfacial tension and extractant packing factor ${ }^{(15)}$ ) and molecular solutions (vertex connectivity, bond probability and cluster distribution). In the bottom row, the microemulsion Winsor II to Winsor III description of third phase formation is contrasted with the proposed percolation transition tied to a liquid-liquid phase transition of a molecular solution. 
In contrast, for an associating molecular solution, aggregation and phase behavior may be interpreted using a graph theoretic approach in which vertices (whether molecules or metal-ligand complexes) are connected by edges which represent intermolecular interactions. As discussed further below and demonstrated for simple liquids, the phase transition for the formation of a dense phase may then be predicted as occurring approximately at a percolation transition for appropriate vertex and connectivity definitions. $\frac{18[20}{20}$ hypothesize here that third phase formation can likewise be approximately identified with a percolation transition for extracted species. Different solvent extraction systems may require different mappings of molecules and their intermolecular interactions to graphical representations. For example, molecular connectivity has been used to determine connectivity in the formation of a system-spanning hydrogen bonded network in the absence of metal ions. ${ }^{[16}$ Such molecular solutions are described by discrete, directional interactions occurring over length scales comparable to that of their molecular constituents. Furthermore, upon the formation of solvate species, the interaction of such solvates may result in solution structure approaching mesoscale lengths. This hierarchical organization of solvates is contrasted with the mesoscale structure of microemulsions where micellar or bicontinuous solutions are characterized by a polar phase stabilized within a nonpolar phase, or vice versa, by an amphiphile. The atomistic resolution provided by MD simulations when combined with a graph theoretical approach can distinguish between the microemulsion and molecular solution paradigms. For example, the hydrogen bonded network of a solution of water-in-oil reverse micelles would show highly connected clusters of a characteristic size related to the reverse micelle length scale. The absence of such features would, instead, indicate a normal molecular solution. 16117 Recent studies have shown that the treatment of organic phases as microemulsions, and the associated colloidal modelling paradigms, has led to counterintuitive or seemingly unphysical conclusions. These include a significant size dependence of the reverse micelles on system variables (e.g., TBP volume fraction or acid concentration) and fitted hard sphere surface attraction values that correspond to the existence of a percolated network of those 
complexes even at low metal concentrations. ${ }^{21}$ These conclusions are presumed to stem from the assumptions used to fit models from experimental scattering data, including, e.g., spherically symmetric interaction potentials that allow for separation of the form and structure factors. However, this interpretation has been contradicted by NMR diffusometry ${ }^{2122}$ and molecular dynamics (MD) simulation studies, $\frac{161172226}{26}$ which find that the organic phase TBP complexes with inorganic aqueous solutes behave as molecular solutions, governed by discrete electrostatic interactions and speciation, rather than as water-in-oil microemulsions. This molecular solution description is also consistent with extended X-ray adsorption fine structure (EXAFS) ${ }^{1127}$ and UV-Vis ${ }^{11}$ spectroscopic data of the uranyl-centered complexes, which suggest a constant coordination environment for the uranyl ion independent of TBP and uranyl concentrations, and is inconsistent with the changing coordination expected in reverse micelles with expanding, highly interconnected polar cores. Given the direct coordination of the uranyl ions by TBP and a relatively low water concentration in the heavy organic phase (roughly one water molecule per three uranyl ions) $\stackrel{13}{ }$ it is reasonable to assume that the uranyl ions do not undergo a substantial structural change in the heavy organic phase such as, e.g., becoming dispersed in a bicontinuous medium. Therefore, we treat this systems as an associating molecular solution whose constituents can form discrete species, including the $(\mathrm{TBP})_{2}\left(\mathrm{UO}_{2}\right)\left(\mathrm{NO}_{3}\right)_{2}$ solvate.

In this study, molecular dynamics simulation is applied to investigate the organic phase association of $(\mathrm{TBP})_{2}\left(\mathrm{UO}_{2}\right)\left(\mathrm{NO}_{3}\right)_{2}$ metal-ligand complexes at high concentration. Prior simulations at low uranyl concentrations identified two modes of uranyl nitrate/extractant complex association in organic solution: an ordered short-range pair and a more isotropic long-range pair. ${ }^{24}$ Importantly, the molecular structure of the extractant's head and tail groups affected those modes of interactions: changing the TBP phosphate head group to a phosphonate group inhibited the short-range ordered dimer while changing the TBP butyl tails to amyl tails suppressed the long-range correlation. Shortening the solvent alkyl chain length from $n$-dodecane to $n$-hexane, or changing the solvent to toluene, or including ex- 
cess TBP and coextracted nitric acid and water all reduced complex association to different degrees. We extend our prior work to simulate the uranium LOC so as to understand higherorder organic phase aggregation and the structural origin of third phase formation for this metal-containing system. Organic phase aggregation of the metal-ligand complexes is quantified using a graph theoretic representation of their self-association, in which the metal-ligand complexes are reduced to single vertices and their association is treated as edges between those vertices. A community analysis of the resulting network then identifies the morphology of the percolating network. We find that in the system studied here, clustering of the metal-ligand complexes yields a predominately linear morphology with "dense subclusters" of highly interconnected vertices. Further, this developed methodology is generalizable to a broad set of chemical systems, where it is possible to vary the definition of a node or edge to elucidate the percolating species responsible for phase separation.

In contrast with colloidal or microemulsion chemistry models, $\stackrel{3}{3}$ our analysis shows a cluster size distribution at the LOC consistent with the percolation theory prediction near the percolation threshold. This is corroborated by the uranium-uranium radial distribution function showing an emerging correlation length consistent with the onset of a percolation phase transition. Therefore, these simulations, and the new graph theoretical analysis method presented, provides evidence that third phase formation in this solvent extraction system is consistent with a liquid-liquid phase transition, where $(\mathrm{TBP})_{2}\left(\mathrm{UO}_{2}\right)\left(\mathrm{NO}_{3}\right)_{2}$ complexes form a system-spanning cluster in solution immediately preceding the organic phase splitting. This approach is further demonstrated to be analogous to simple binary mixtures where the minor component percolates with increasing concentration immediately before reaching the liquid-liquid coexistence curve. $\underline{1819}$

In summary, this study proposes a methodology for predicting third phase formation via a graph theoretical formalism where the node and edge definitions enable identification of the percolated network at the onset of the phase transition for the appropriate vertex and edge definitions. This work creates a framework for elucidating the appropriate models 
used to describe phase transitions of multicomponent solutions and also a paradigm for understanding how molecular-scale detail influence macroscopic phase transition behavior.

\section{Methodology}

\section{Simulation Force Fields}

The force fields used in this study are reported in detail in the Supporting Information of ref. 24. The modeling approach is summarized here. Simulation times of many 10s of ns are required to equilibrate and sample the slow dynamics of organic phase metal-ligand complex association, necessitating the use of classical, additive potentials. The stoichiometry of the uranyl nitrate/TBP metal-ligand complexes is monodisperse and independent of organic phase uranium concentration. $\frac{1127}{227}$ Rather than the five-fold coordination found in aqueous solutions, the organic phase uranyl metal center of the $(\mathrm{TBP})_{2} \mathrm{UO}_{2}\left(\mathrm{NO}_{3}\right)_{2}$ complex is six-fold coordinate with the nitrates binding in a bidentate manner. For this system, we thus constrained the nitrate positions to enforce the bidentate coordination. To account for charge transfer, partial atomic charges for the uranyl and nitrate ions were derived from density functional theory electronic structure calculations of the assembled complex, rather than using integer formal charges of separated ions. Force field parameters for n-dodecane and TBP were modified from the general AMBER force field (GAFF) ${ }^{28}$ to reproduce physicochemical properties of TBP/n-dodecane mixtures including TBP molecular dipole, density, enthalpy of mixing and volume change on mixing. ${ }^{29}$ Nitric acid was modeled with GAFF ${ }^{28}$ parameters and water with the TIP4P model. 30

\section{Simulation Methodology}

Although direct simulation of a two to three phase transition is desirable, it is not generally achievable over simulation-accessible time scales. Grand canonical ensemble simulation of a light organic phase in chemical equilibrium with a heavy phase is also challenging for this 
system due to ambiguities in the structure of the metal-ligand complex in the heavy organic phase. For example, Chiarizia et al. found that while the coordination geometry of the metal-ligand complexes is largely unchanged between the organic phase at the LOC and the heavy organic phase, a minority of nitrate anions change to monodentate coordination. 11 Given these practical considerations, we identify the onset of organic phase splitting from a monophasic simulation of the organic phase at the uranium LOC.

The equilibrated cubic simulation box was $13.37 \mathrm{~nm}$ on each side, with a composition of $320 \mathrm{UO}_{2}\left(\mathrm{NO}_{3}\right)_{2}, 1048$ total TBP (free and uranyl-bound), 4832 n-dodecane, $176 \mathrm{H}_{2} \mathrm{O}$ and $392 \mathrm{HNO}_{3}$. Molecular concentrations were obtained from ref. 13. The composition gives 0.26 M uranyl nitrate, corresponding to the LOC for $20 \%$ volume TBP in n-dodecane for uranyl extraction from $10 \mathrm{M}$ aqueous phase nitric acid. The initial configuration was generated with Packmol. ${ }^{31}$ The assembled $(\mathrm{TBP})_{2}\left(\mathrm{UO}_{2}\right)\left(\mathrm{NO}_{3}\right)_{2}$ complexes were distributed randomly into the n-dodecane. "Free" TBP (not bound to uranyl nitrate) and coextracted water and nitric acid are also distributed randomly in the initial configuration.

Simulations were conducted using the GROMACS 4.5.5 software package. ${ }^{32}$ Simulations were conducted in the isobaric isothermal NPT ensemble using periodic boundary conditions. Pressure was set to 1 bar with the Parinello-Rahman barostat $\frac{33}{3}$ using a 2 ps time constant and the temperature was set to $300 \mathrm{~K}$ with the Nosé-Hoover thermostat $\stackrel{34}{34}$ using a 0.2 ps time constant. The leap-frog Verlet integrator was implemented with a 2 fs time step and the LINCS algorithm $\frac{35}{35}$ to constrain hydrogen-containing bonds. A $1.5 \mathrm{~nm}$ cut-off was used for Lennard-Jones and short range electrostatic interactions with Particle-Mesh Ewald summation for long range electrostatic interactions. The simulation ran for $145 \mathrm{ns,}$ equilibrating over the first $35 \mathrm{~ns}$, and sampled for analysis at 20 ps intervals.

\section{Graph Theoretic Analysis of $(\mathrm{TBP})_{2}\left(\mathrm{UO}_{2}\right)\left(\mathrm{NO}_{3}\right)_{2}$ Association}

The analysis workflow for this study, with the relationships between inputs, intermediate analyses and results are presented as a flowchart in Figure 2. Interactions of $(\mathrm{TBP})_{2}\left(\mathrm{UO}_{2}\right)\left(\mathrm{NO}_{3}\right)_{2}$ 
complexes are first quantified using a graph theoretic approach. Each complex is defined as a single vertex. An edge is considered to exist between vertices if the distance between the uranium atoms of those corresponding complexes is no more than $1.5 \mathrm{~nm}$. The cutoff distance was chosen from the approximate minimum in the uranium-uranium radial distribution function. Clusters are defined as connected components of the graph containing all of the vertices. The largest cluster, containing the most vertices, is identified at each time step.
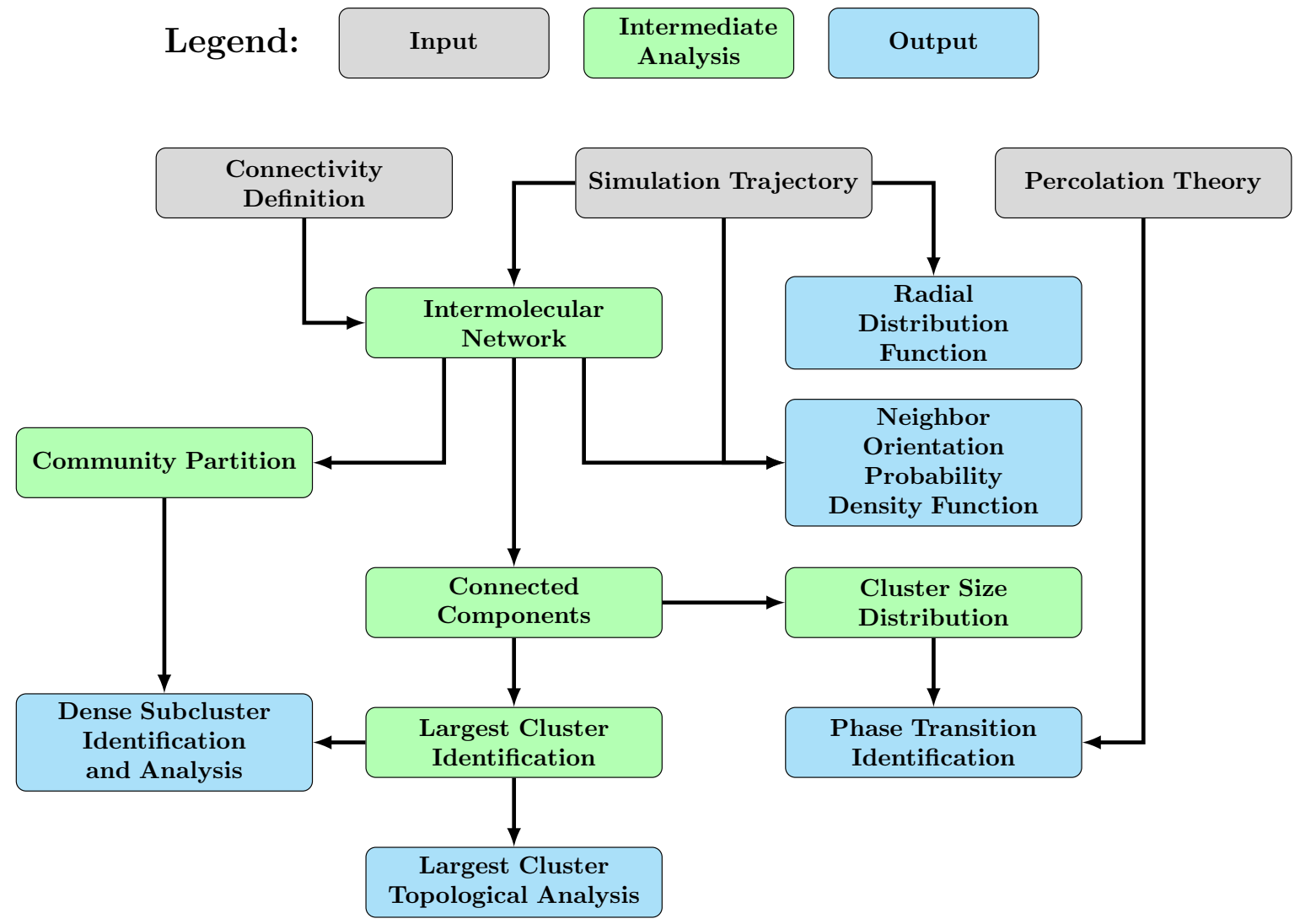

Figure 2: Workflow for inputs (gray nodes), intermediate analysis steps (green nodes) and results (blue nodes) are illustrated as a flowchart. 


\section{Results}

\section{Uranium-Uranium Correlation}

Aggregation of the $(\mathrm{TBP})_{2}\left(\mathrm{UO}_{2}\right)\left(\mathrm{NO}_{3}\right)_{2}$ complexes is analyzed through the radial distribution function $(\mathrm{RDF}), g(r)$, between pairs of uranium atoms. The uranium-uranium RDF is plotted in Figure $3 \mathrm{~A}$ for the 10-35 ns and 120-145 ns time ranges of the trajectory. The uranium-uranium correlation takes shape over the first $10 \mathrm{~ns}$ of the trajectory, showing the short-range dimer peak near $0.6 \mathrm{~nm}$ and the long-range peak beyond $1 \mathrm{~nm}$, as previously reported at low uranyl concentration. $\stackrel{24}{24}$ However, the formation of extended clusters in this concentrated system is evidenced by the emergence of uranium-uranium correlations beyond those short-range and long-range pairs (Figure $3 \mathrm{~A}$ inset). While the uranium-uranium $g(r)$ is uncorrelated for distances beyond $2 \mathrm{~nm}$ for the 10-35 ns range, $g(r)$ values greater than unity are observed after the system has equilibrated. The linear $g(r)$ behavior in the 120-145 ns time range between 2 and $5 \mathrm{~nm}$ indicates solution heterogeneity resulting from uranium-rich and uranium-poor domains in solution. $\underline{36}$

\section{Metal-Ligand Clustering and Percolation}

The appearance of a third phase is a result of the favorable association of solutes. Under appropriate conditions, that association leads to the formation of larger clusters that eventually become a percolating (system-spanning) cluster. Then, at some point the heavily connected phase becomes thermodynamically favorable. While related, these are distinct events. Percolation in ideal lattices is a second-order phase transition, while the appearance of a new thermodynamically stable phase is a first-order phase transition (away from the critical point). For non-ideal systems with interactions, percolation has also been associated with the spinodal. $\frac{18 \mid 19}{19}$ Nonetheless, it has been shown that the percolation point can closely track the thermodynamic phase boundary. For example, the miscibility gap in simple liquids can be identified with percolation theory: $\frac{37}{37}$ water undergoes a percola- 

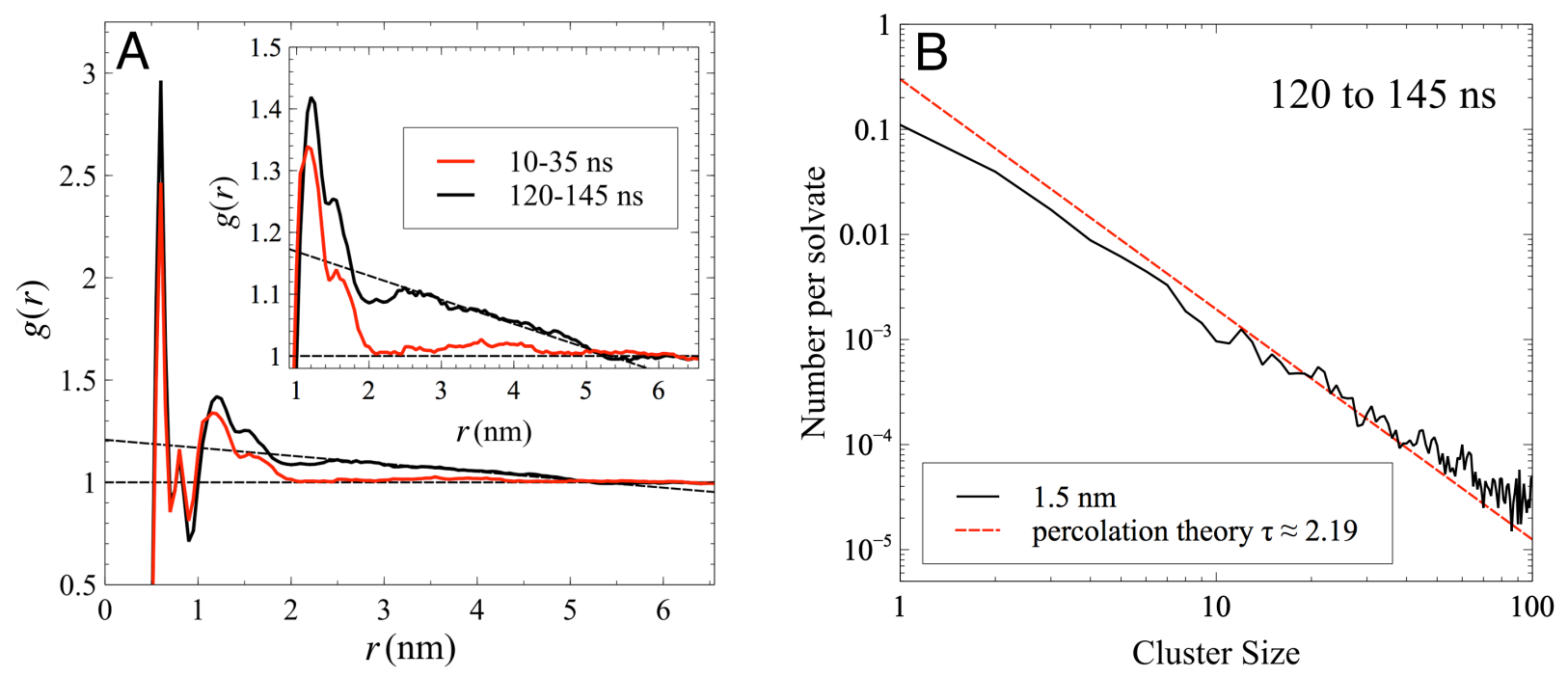

Figure 3: In panel A, the uranium-uranium RDF is plotted for two different time ranges of the trajectory with the long-range correlation between 2 and $5 \mathrm{~nm}$ uranium-uranium distances highlighted in the inset. Dashed lines are drawn to illustrate the linear slope of $g(r)$ at long distances. In panel B, the $(\mathrm{TBP})_{2}\left(\mathrm{UO}_{2}\right)\left(\mathrm{NO}_{3}\right)_{2}$ complex cluster size distribution using a $1.5 \mathrm{~nm}$ uranium-uranium distance cutoff is plotted for the 120-145 ns time range of the trajectory. The theoretical power law distribution at the percolation threshold given by the Fisher exponent for three dimensions, $\tau \approx 2.19$, is plotted as a dashed red line for comparison. 
tion transition upon increasing its concentration in tetrahydrofuran immediately preceding the phase boundary. $\frac{18 \mid 19}{}$ Previously, we connected the third phase formation phase boundary in the $\mathrm{TBP} / \mathrm{HNO}_{3} / \mathrm{H}_{2} \mathrm{O} / \mathrm{n}$-dodecane system to the emergence of a percolated hydrogen bonded network. In the system studied here, there is neither a percolated hydrogen bonded network nor the formation of water-in-oil micelles with interconnected cores of $\mathrm{H}_{2} \mathrm{O}, \mathrm{HNO}_{3}$ and $\mathrm{UO}_{2}\left(\mathrm{NO}_{3}\right)_{2}$ molecules. Rather, we apply the graph theoretical description of solute association to the $(\mathrm{TBP})_{2}\left(\mathrm{UO}_{2}\right)\left(\mathrm{NO}_{3}\right)_{2}$ complexes themselves. While the solution retains its molecular character, the dominant attractive interaction which drives phase separation occurs between $(\mathrm{TBP})_{2}\left(\mathrm{UO}_{2}\right)\left(\mathrm{NO}_{3}\right)_{2}$ solvates. The relative stability of that species means a practical description of the solution may consider interactions between discrete solvates rather than individual molecules. However, the short length scale of these solvates necessitates a molecular description of their geometry and interaction, precluding a micellar structure where an interface forms between two distinct phases.

The graph theoretical analysis of the phase transition allows molecular level characterization of molecular association which can distinguish a microemulsion from a molecular solution. Additionally, it allows investigation of the percolation transition and its relationship to the appearance of the third phase. However, unlike lattices, the definition of continuum connectivity for MD simulations is not unique. Even for a well-defined interaction, such as a hydrogen bond, the choice of cut-off, whether energetic or geometric, is arbitrary. For this system, we define solvate connectivity of nearest neighbors based on a distance cut-off obtained from the U-U RDF.

At the percolation threshold, the mean cluster size of the percolating component diverges as a cluster of infinite size exists with a finite probability. In finite systems, as is the case for molecular simulation, that infinite cluster manifests as a system-spanning cluster. The cluster size distribution transitions from an exponential distribution far from the percolation threshold to a power law distribution in the neighborhood of the threshold. The power law distribution of cluster sizes is given by $n_{s} \sim s^{-\tau}$ where $n_{s}$ is the fraction of clusters of size $s$ 
and $\tau$ is the Fisher exponent having a value of $\approx 2.19$ in three dimensions. $\frac{38}{38}$

Signatures of percolation, such as the power law cluster size distribution, are readily accessible from simulation and provide a means to identify the liquid-liquid phase boundary for systems where it may not be possible to directly simulate phase separation under practical simulation length and time scales. We tested the applicability of this approach for a binary Lennard-Jones (L-J) fluid model system with inter-particle connectivity more directly analogous to the solvate connectivity defined here. We observe the same qualitative behavior for the L-J fluid as for the water/tetrahydrofuran mixture, supporting the broader utility of continuum percolation as a means of approximate demarcation of the two phase region. Simulation details and schematic phase diagram with simulation results for the L-J fluid are provided in the Supporting Information.

This method is applied to the liquid-liquid phase boundary of the uranyl nitrate solution at the LOC. The (TBP $)_{2}\left(\mathrm{UO}_{2}\right)\left(\mathrm{NO}_{3}\right)_{2}$ complex cluster size distributions on a log-log scale for the 120-145 ns time range is nearly equal to the theoretical cluster size distribution at the percolation threshold, $\tau$, as shown in Figure $3 \mathrm{~B}$. In conjunction with the increasing correlation length observed in the uranium-uranium RDFs - another property which diverges at the percolation threshold - these results are consistent with the formation of an incipient "infinite" (TBP $)_{2}\left(\mathrm{UO}_{2}\right)\left(\mathrm{NO}_{3}\right)_{2}$ solvate cluster expected to precede third phase formation.

\section{Relative Orientation of Associating Metal-Ligand Complexes}

The spherical anisotropy resulting from the polar and aliphatic regions of the exposed surface of the (TBP $)_{2}\left(\mathrm{UO}_{2}\right)\left(\mathrm{NO}_{3}\right)_{2}$ complexes may impact the morphology of their clustering in solution. The relative orientation of metal-ligand complex pairs is quantified by the angle between $\mathrm{P}_{1}-\mathrm{U}_{1}$ and $\mathrm{U}_{1}-\mathrm{U}_{2}$ vectors of associating complexes. Here, the subscript number refers to the two different metal-ligand complexes. This angle, illustrated in Figure 4 , is measured for the four phosphorus atoms of each pair of neighboring complexes. Due to the position of the two $\mathrm{P}$ atoms in one complex to be on opposite sides of the $\mathrm{U}$ center, and the 

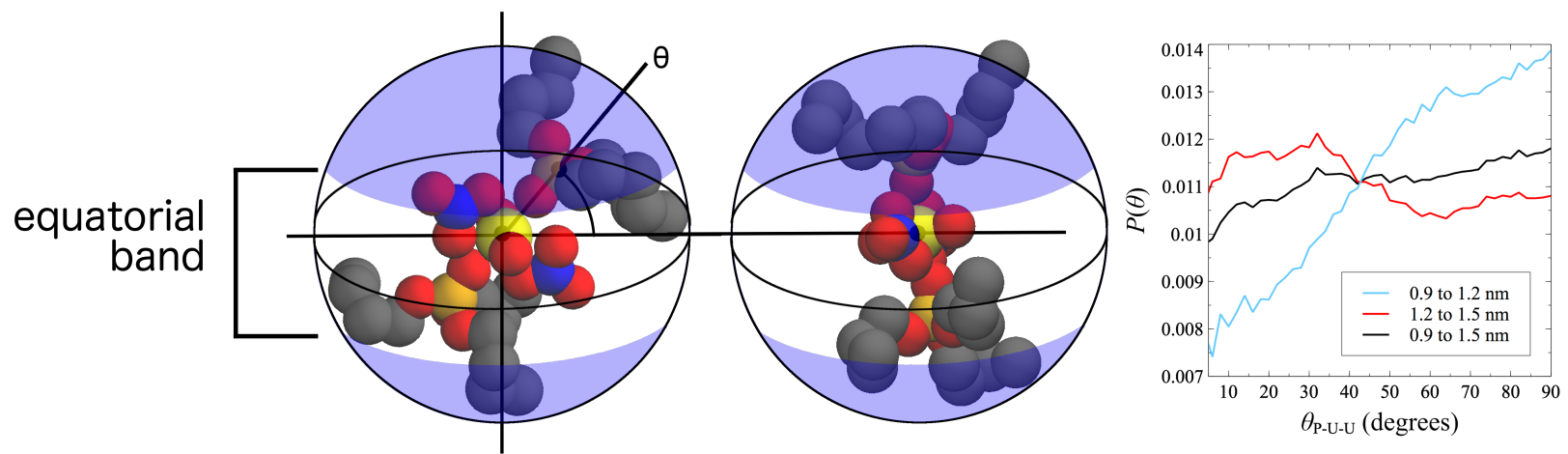

Figure 4: The relative orientation of the metal-ligand complex, $\theta$, is measured by the angle between the intra-complex phosphorus-uranium and inter-complex uranium-uranium vectors. The preference for values between $60^{\circ}$ and $90^{\circ}$ at close U-U distances results in an "equatorial band" region of the complex-illustrated by shading the rest of the spherewhich are preferentially exposed to neighboring complexes. Oxygen atoms are drawn in red, nitrogen in blue, carbon in gray, phosphorus in orange and uranium in yellow. On the right, the normalized probability density functions, $\mathrm{P}(\theta)$, are plotted in black, blue and red lines for metal-ligand complex pairs from the 120-145 ns time range with uranium-uranium distances of 0.9 to $1.5 \mathrm{~nm}, 0.9$ to $1.2 \mathrm{~nm}$, and 1.2 to $1.5 \mathrm{~nm}$, respectively.

resulting symmetry around $\theta=90^{\circ}$, values over $90^{\circ}$ are reflected across $\theta=90^{\circ}$. The resulting probability density functions for that angle are plotted in Figure 4 for three uranium-uranium distance ranges. Probability densities are normalized by $\sin (\theta)$ to account for the solid angle dependence on $\theta$. Note that the normalized curves satisfy the normalization condition $\int_{0}^{\pi / 2} P(\theta) \sin \theta d \theta=1$, and a constant value of $P(\theta)$ would correspond to a random distribution of orientations of one cluster relative to the direction to the other cluster.

Solvate pairs have an increased orientation probability for $\theta$ values close to $90^{\circ}$ at closer $\mathrm{U}-\mathrm{U}$ distances, shown by the 0.9 to $1.2 \mathrm{~nm}$ distance range in Figure 4 . This is attributed to the region of the exposed solvate surface with polar molecules - the uranyl apical oxygens and nitrates, rather than the nonpolar alkyl tails of the TBP - at that orientation, as illustrated in Figure 4 by the "equatorial band." Conversely, at longer U-U distances (1.2 to $1.5 \mathrm{~nm}$ ), the probability density is flatter with slightly increased probabilities for the opposite orientation: the nonpolar alkyl tails of the coordinating TBP molecules orient towards the other solvate. This could result from less efficient packing of the complexes with the solvent at that U-U distance and relative orientation. The observed preferential orientations of the metal-ligand 
complexes imply a dependence of the long-range pair and resulting clustering morphology on the presence of the actinyl apical oxygens and the nitrate counterions, as well as the extractant alkyl tail length and branching or lack thereof.

\section{Morphological Features of Metal-Ligand Complex Clusters}

The morphology of the extended clustering preceding third phase formation is investigated by considering the topology of the largest cluster, which becomes the system spanning cluster that emerges at the percolation threshold for a finite system. A representative largest cluster from time $=125 \mathrm{~ns}$ is depicted in Figure 5 as both a graph representation and as a snapshot. The complexes are drawn as spheres of diameter $1.5 \mathrm{~nm}$ corresponding to their connectivity cutoff with those not in the largest cluster drawn with partial transparency. The largest cluster contains, on average, $96 \pm 38$ of the 320 total vertices in the graph. The large variability of the largest cluster size stems from the dynamic equilibrium between the systemspanning and smaller, finite sized clusters in solution.

Two primary characteristics of the largest cluster topology are apparent from the graphical representation: the graph is largely linear without substantial crosslinking and there are relatively small "dense subclusters" of highly interconnected vertices within the total cluster. The linear character of the large clusters could result from preferred complex coordination at the polar band, which provides more favorable orientations for a third complex to directly associate with only one of those two complexes, forming a linear triplet. The relatively weak association of the long-range pairs appears to not enforce a particular structural motif within the extended cluster beyond the dense subclusters.

To systematically identify dense subclusters within the largest cluster at each snapshot, we partition the largest cluster connected component into "communities," defined to maximize connectivity within the communities while minimizing connectivity between them. The modularity optimization algorithm ${ }^{39}$ implemented to compute the community partitions is described in the Supporting Information. Once the community partition is determined, we 


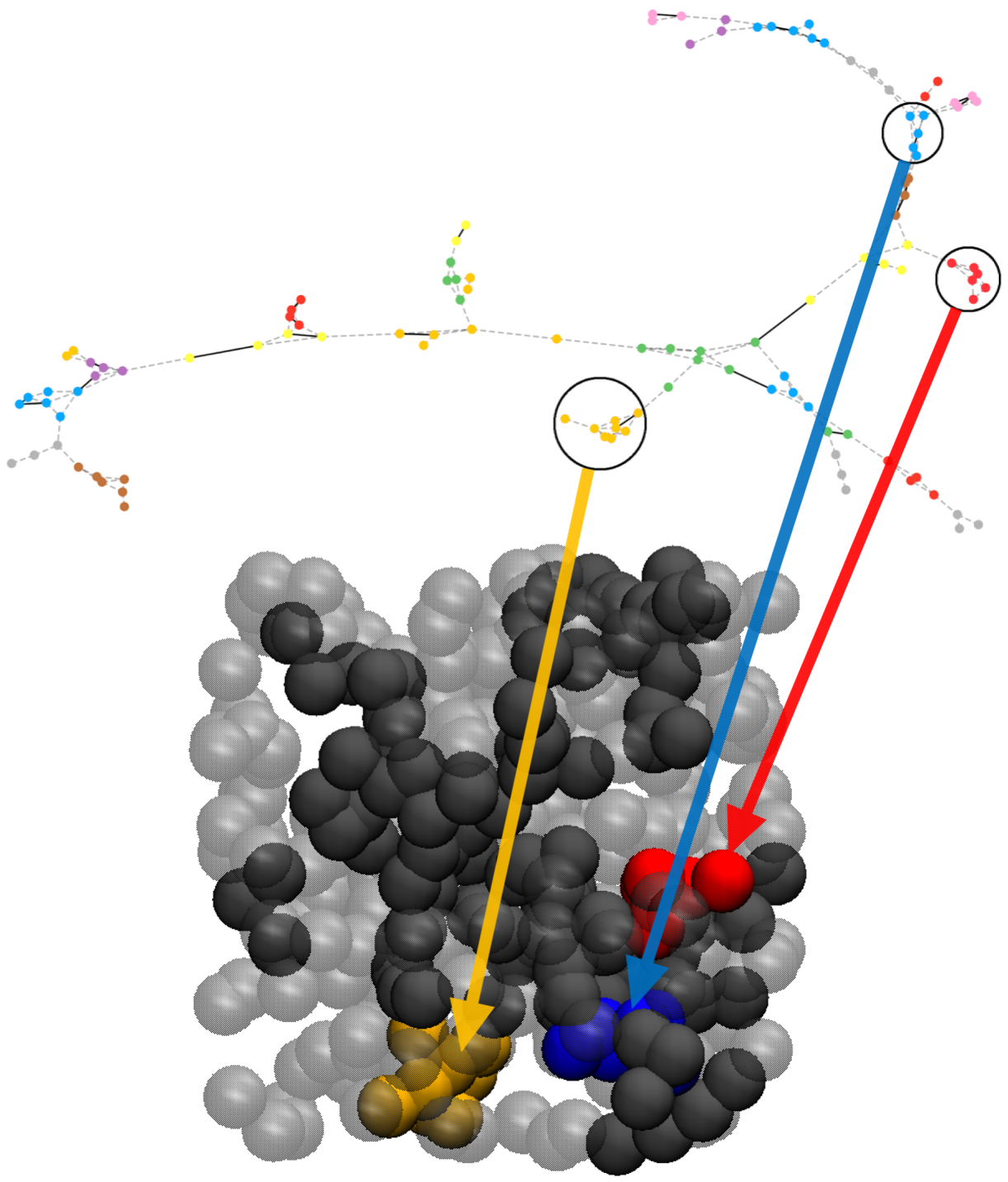

Figure 5: Graphical representation of a largest cluster (top) at time $=125 \mathrm{~ns}$ with colors indicating community membership (colors are reused between unconnected communities). Short-range pair edges are drawn with solid lines while long-range pair edges are drawn with dashed lines. Of the 28 communities, 3 are identified as "dense subclusters" and circled. Snapshots of the periodic simulation cell (bottom) from which the graph is computed depict the metal-ligand complexes as spheres of diameter $1.5 \mathrm{~nm}$. Metal-ligand complexes in the largest cluster are colored in dark gray with remaining complexes drawn with partial transparency. The three dense subclusters are colored according to their coloring on the community color-coded graph. Note that the length of edges and vertex positions do not correspond to any physical lengths or positions, and are chosen to aid ease of visualizing the graph connectivity. 
define dense subclusters as communities having a ratio of the number of edges within the community to the total number of vertices comprising that community of greater than 1.5. The distribution of number of internal edges per node for each community, given in the Supporting Information, showed several peaks and this criterion was chosen to isolate the most densely connected region of the distribution. Using this metric, we identify the dense subclusters which form in the largest cluster at each time step. For the time step illustrated in Figure 5, the three dense subclusters determined from the 28 total communities of that cluster are highlighted. Between 10 and 35 ns, on average there were 1.8 dense subclusters per snapshot with an average size of 7.5 vertices. By contrast, between 120 and 145 ns there were 2.2 dense subclusters per snapshot having an average of 6.9 vertices. Separately considering short-range pair edges from long-range pairs, as illustrated in Figure 5 by solid and dashed lines, respectively, the number of short-range edges per vertex within dense subclusters divided by the number of short-range edges per vertex over the entire largest cluster was 1.54 for 10-35 ns and 1.42 for 120-145 ns (as compared to that same ratio for all edges of 1.34 and 1.29 for those time ranges, respectively). Therefore, short-range pairs are found disproportionately within those dense subclusters. System variables which impact short-range pair formation, such as extractant head group moeity, ${ }^{24}$ are therefore expected to affect the degree of dense subcluster formation within the largest cluster. Reducing the community definition ratio to greater than 1.0 instead of 1.5 substantially increases the total number of dense subclusters, but similarly shows a short-range edge ratio of 1.29 averaged over both 10-35 and 120-145 ns time ranges (compared to that ratio for all edges of 1.07).

The outlined method for dense subcluster identification is flexible, both in the community identification algorithm, its ability to accommodate weighted graphs and in the criterion used to identify those subclusters from the community partition. This approach to partitioning solute networks can be applied to a wide range of solvent extraction organic phases where, generally, domains of strongly interconnected polar solutes form extended networks under high solute concentrations. To further characterize clustering of the complexes and their 
time evolution during equilibration, three standard graph topological properties qualitatively measuring compactness and connectivity are plotted in Figure 6 for the largest cluster at 200 ps intervals: the number of edges per vertex, the global clustering coefficient and the graph link efficiency. The edges per vertex is the ratio of total number of edges to the total number of vertices. The global clustering coefficient is the fraction of vertex triplets which are closed, i.e., all vertices in the triplet are connected to all other vertices. The graph link efficiency is defined as one minus the mean graph distance divided by the total number of edges. The graph distance is the shortest number of edges connecting a given pair of vertices, with the mean obtained over all the vertex pairs. First or second order polynomial fits are overlayed on the data to highlight time evolution of these properties during equilibration $(0$ to $35 \mathrm{~ns}$ ) and over the remainder of the trajectory (35 to $145 \mathrm{~ns}$ ). For all three properties, their values increase rapidly during the initial $35 \mathrm{~ns}$. Then, after $35 \mathrm{~ns}$, slow relaxation of the largest cluster results in slight decreases in all three properties.
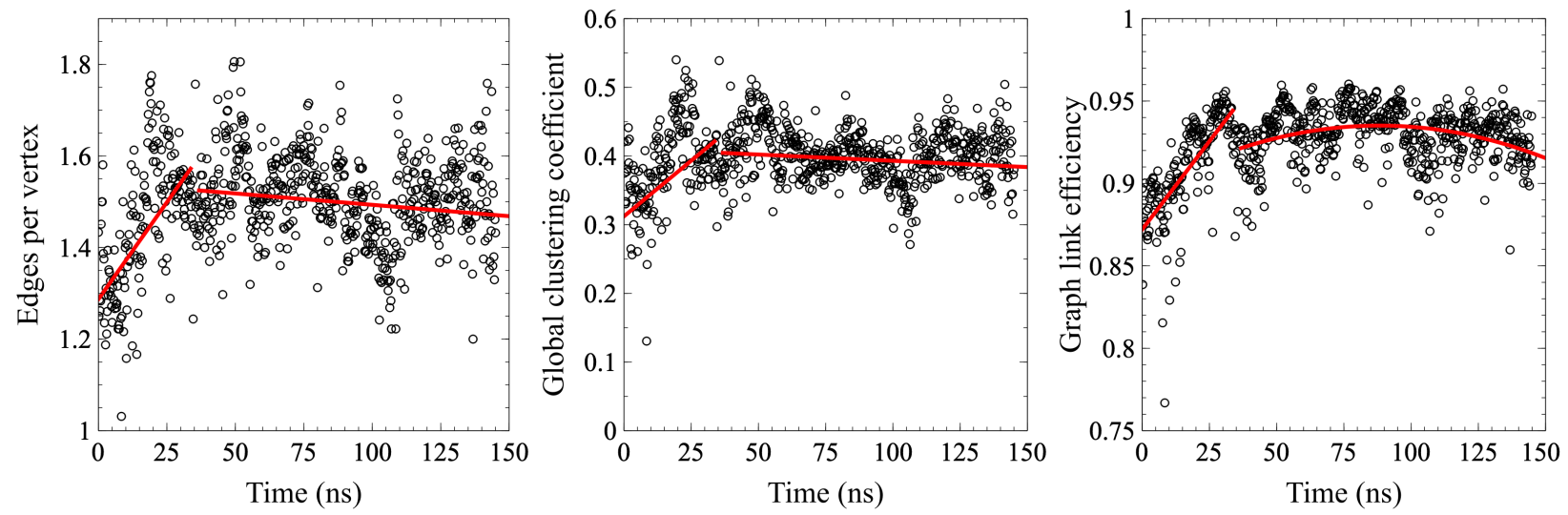

Figure 6: The edges per vertex (left), global clustering coefficient (center) and graph link efficiency (right) are plotted for the largest cluster as a function of simulation time at 200 ps intervals. Data is plotted in black circles. Polynomial fits are overlayed in red lines for two trajectory time ranges as guides to the eye to illustrate trends of the properties during the trajectory.

The clustering topology of the metal-ligand complexes bears similarities to simpler models which have been applied to liquids and for which percolation phase transitions are observed: the cubic lattice and the Baxter adhesive hard sphere. Here, we briefly comment on the 
qualitative similarities and differences between the $(\mathrm{TBP})_{2}\left(\mathrm{UO}_{2}\right)\left(\mathrm{NO}_{3}\right)_{2}$ simulation results and those theoretical models. As illustrated in the Supporting Information, the largest cluster on a cubic lattice near the bond percolation threshold shows a similar linear, branching cluster morphology as the simulation data presented here. However, vertex clusters do not readily form on a cubic lattice due to the connectivity of that lattice. The dense subclusters observed in this study are qualitatively similar to the "dense subunits" described by Miller and Frenkel ${ }^{40}$ from Monte Carlo simulation of Baxter adhesive hard spheres. There, subclusters form within a percolating network to maximize the energetically favorable contacts between neighboring spheres. However, we expect the graph of the system-spanning cluster for Baxter adhesive hard spheres to be, in general, more compact and interconnected than the $(\mathrm{TBP})_{2}\left(\mathrm{UO}_{2}\right)\left(\mathrm{NO}_{3}\right)_{2}$ graph we have observed here.

\section{Conclusions}

Third phase formation is an empirically well-documented phenomenon in liquid/liquid extraction. However, it eludes a description linking molecular-level interactions to the resulting macroscopic phase behavior. Using atomistic simulation, and incorporating the experimental understanding of the structure of $(\mathrm{TBP})_{2}\left(\mathrm{UO}_{2}\right)\left(\mathrm{NO}_{3}\right)_{2}$ adducts in solution, we study association of those metal-ligand complexes at the uranyl LOC. We find the third phase formation phase transition in this system is consistent with a solvate percolation mechanism, wherein the complexes form a system-spanning network as the system nears the phase boundary. This mechanism is analogous to percolation of the minor component of a binary liquid mixture which is observed when approaching the liquid-liquid coexistence curve from the single phase to the two phase region. $\frac{18}{20141}$

Complex self-association and resulting aggregation morphology were characterized. Orientational preferences were observed between associating complexes wherein the complexes were more likely to present their "equatorial band" region to neighboring complexes within 
the long-range pairs. Clusters of associating complexes were quantified and the morphology, connectivity and compactness of the largest cluster, being the precursor to the percolated system-spanning cluster, was quantified using graph theoretical metrics. The cluster was partitioned into communities, with an intra-community connectivity threshold applied to identify "dense subclusters" within the mostly linear solvate network. Short-range complex pairs were found to disproportionately populate those dense subclusters, implying a sensitivity of aggregate morphology to system variables which affect short-range pair formation. ${ }^{24}$

Recent studies have proposed a diversity of mechanisms for aggregation and third phase formation across a variety of solvent extraction systems. $\frac{1611725 \mid 42}{162}$ Those studies highlight the role of molecular-scale interactions, often different between systems, in governing organic phase aggregation. The method presented here for identifying the onset of third phase formation is independent of the specific type of molecular connectivity. The appropriate choices of vertices and edges may differ between systems depending on their underlying molecular-scale interactions. Previously, we proposed that third phase formation in the TBP $/ \mathrm{H}_{2} \mathrm{O} / \mathrm{HNO}_{3}$ system in the absence of metal ions resulted from percolation of the hydrogen bonded network consisting of those molecules at high extracted water and acid concentrations. ${ }^{16}$ In this study, given the role of coordinating metal ions, we do not observe a percolated network of only hydrogen bonds, although TBP in excess of the uranyl nitrate complexes and coextracted water and nitric acid are present. Rather, the percolation is characterized directly in terms of $(\mathrm{TBP})_{2}\left(\mathrm{UO}_{2}\right)\left(\mathrm{NO}_{3}\right)_{2}$ solvate pairing.

The analysis methodology for solvate coarse graining and cluster partitioning to quantify hierarchical organization within the solution could be broadly applied to complex organic phases. Treating third phase formation as a liquid-liquid phase transition of a multicomponent associating molecular solution, and using an appropriate graph theoretic description of solute interactions, has enabled simulation prediction of the phase boundary. The extension of this approach and its implications to other solvent extraction systems will require further investigation, possibly through experimental quantification of critical behavior near the 
phase boundary. $\frac{43}{4}$ The interpretation of organic phase aggregation and third phase formation outlined in this study may explain how system variables such as solvent or extractant molecular structure ${ }^{\sqrt{24}}$ promote or inhibit third phase formation. If simple models, such as lattice percolation, $\stackrel{44}{ }$ can sufficiently describe aggregation in solvent extraction systems, they could enable high-throughput phase diagram development.

\section{Conflicts of interest}

There are no conflicts of interest to declare.

\section{Acknowledgements}

This work was supported by the Nuclear Regulatory Commission Faculty Development Award NRC-HQ-11-G-38-0062 and by the Department of Energy Basic Energy Sciences Separations program grant DE-SC0001815. We acknowledge the Colorado School of Mines High Performance Computing center for computational resources.

\section{References}

(1) Cinar, S.; Cinar, H.; Chan, H. S.; Winter, R. Journal of the American Chemical Society 2019, 141, 7347-7354.

(2) Le Ferrand, H.; Duchamp, M.; Gabryelczyk, B.; Cai, H.; Miserez, A. Journal of the American Chemical Society 2019, 141, 7202-7210.

(3) Testard, F.; Zemb, T.; Bauduin, P.; Berthon, L. Ion Exchange and Solvent Extraction: A Series of Advances; CRC Press: Boca Raton, 2009; Vol. 19; Chapter Third-Phase Formation in Liquid/Liquid Extraction: A Colloidal Approach, pp 381-428. 
(4) Rydberg, J.; Cox, M.; Musikas, C.; Choppin, G. Solvent Extraction Principles and Practices, 2nd ed.; Marcel Dekker: New York, 2004.

(5) Rao, P.; Kolarik, Z. Solvent Extraction and Ion Exchange 1996, 14, 955-993.

(6) Nash, K.; Braley, J. C. Challenges for actinide separations in advanced nuclear fuel cycles; American Chemical Society, 2010; Chapter Nuclear Energy and the Environment, pp 19-38.

(7) Duvail, M.; van Damme, S.; Guilbaud, P.; Chen, Y.; Zemb, T.; Dufrêche, J. Soft Matter 2017, 13, 5518-5526.

(8) Spadina, M.; Bohinc, K.; Zemb, T.; Dufrêche, J. Langmuir 2019, 35, 3215-3230.

(9) Karmakar, A.; Duvail, M.; Bley, M.; Zemb, T.; Dufrêche, J. Colloids and Surfaces A 2018, 555, 713-727.

(10) Zemb, T.; Bauer, C.; Bauduin, P.; Belloni, L.; Dèjugnat, C.; Diat, O.; Duboi, V.; Dufrêche, J.; Dourdain, S.; Duvail, M.; Larpent, C.; Testard, F.; Pellet-Rostaing, S. Colloid and Polymer Science 2015, 293, 1-22.

(11) Chiarizia, R.; Jensen, M.; Borkowski, M.; Ferraro, J.; Thiyagarajan, P.; Littrell, K. Solvent Extraction and Ion Exchange 2003, 21, 1-27.

(12) Chiarizia, R.; Jensen, M.; Borkowski, M.; Ferraro, J.; Thiyagarajan, P.; Littrell, K. Separation Science and Technology 2003, 38, 3313-3331.

(13) Chiarizia, R.; Nash, K.; Jensen, M.; Thiyagarajan, P.; Littrell, K. Langmuir 2003, 19, 9592-9599.

(14) Nave, S.; Mandin, C.; Martinet, L.; Berthon, L.; Testard, F.; Madic, C.; Zemb, T. Physical Chemistry Chemical Physics 2004, 6, 799-808.

(15) Isrealachvili, J. Colloids and Surfaces A 1994, 91, 1-8. 
(16) Servis, M.; Wu, D.; Braley, J. Physical Chemistry and Chemical Physics 2017, 19, $11326-11339$.

(17) Mu, J.; Motokawa, R.; Akutsu, K.; Nishitsuji, S.; Masters, A. Journal of Physical Chemistry B 2018, 122, 1439-1452.

(18) Oleinikova, A.; Brovchenko, I.; Geiger, A.; Guillot, B. Journal of Chemical Physics 2002, 117, 3296-3304.

(19) Pártay, L.; Jedlovszky, P.; Brovchenko, I.; Oleinikova, A. Physical Chemistry and Chemical Physics 2007, 9, 1341-1346.

(20) Misawa, M.; Yoshida, K. Journal of the Physical Society of Japan 2000, 69, 3308-3314.

(21) Baldwin, A.; Servis, M.; Yang, Y.; Bridges, N.; Wu, D.; Shafer, J. Journal of Molecular Liquids 2017, 246, 225-235.

(22) Baldwin, A.; Yang, Y.; Bridges, N.; Braley, J. Journal of Physical Chemistry B 2016, 120, 12184-12192.

(23) Ivanov, P.; Mu, J.; Leay, L.; Sharrad, C.; Masters, A. Solvent Extraction and Ion Exchange 2017, 35, 251-265.

(24) Servis, M. J.; Wu, D. T.; Shafer, J. C.; Clark, A. E. Chemical Communications 2018, 1, 10064-10067.

(25) Motokawa, R.; Kobayashi, T.; Endo, H.; Mu, J.; Williams, C. D.; Masters, A. J.; Antonio, M. R.; Heller, W. T.; Nagao, M. ACS Central Science 0, 0, null.

(26) Guilbaud, P.; Berthon, L.; Louisfrema, W.; Diat, O.; Zorz, N. Chemistry: A European Journal 2017, 23, 16660-16670.

(27) Den Auwer, C.; Lecouteux, C.; Charbonnel, M.; Madic, C.; Guillaumont, R. Polyhedron 1997, 16, 2233-2238. 
(28) Wang, J.; Romain, M.; Caldwell, J.; Kollman, P.; Case, D. Journal of Computational Chemistry 2004, 25, 1157-1174.

(29) Servis, M.; Wu, D.; Shafer, J. Journal of Molecular Liquids 2018, 253, 314-325.

(30) Jorgensen, W. L.; Chandrasekhar, J.; Madura, J. D.; Impey, R. W.; Klein, M. L. Journal of Chemical Physics 1983, 79, 926-935.

(31) Martinez, L.; Andrade, R.; Birgin, E.; Martinez, J. Journal of Computational Chemistry 2009, 30, 2157-2164.

(32) Hess, B.; Kutzner, C.; Spoel, D.; Lindhal, E. Journal of Chemical Theory and Computation 2008, 4, 435-447.

(33) Parrinello, M.; Rahman, A. Journal of Applied Physics 1981, 52, 7182-7190.

(34) Hoover, W. Physical Review A 1985, 31, 1695-1697.

(35) Hess, B.; Bekker, H.; Berendsen, H.; Fraaije, J. Journal of Computational Chemistry 1997, 18, 1463-1472.

(36) He, S.; Maibaum, L. Journal of Physical Chemistry B 2018, 122, 3961-3973.

(37) Klein, W. Physical Review Letters 1990, 65, 1462-1465.

(38) Lorenz, C. D.; Ziff, R. M. Physical Review E 1998, 57, 230-236.

(39) Blondel, V. D.; Guillaume, J. L.; Lambiotte, R.; Lefebvre, E. Journal of Statistical Mechanics: Theory and Experiment 2008, P10008.

(40) Miller, M.; Frenkel, D. Journal of Physics: Condensed Matter 2004, 16, 4901-4912.

(41) Campi, X.; Krivine, H.; Sator, N. Physica A 2001, 296, 24-30.

(42) Antonio, M.; Ellis, R.; Estes, S.; Bera, M. Physical Chemistry and Chemical Physics 2017, 19, 21304-21316. 
(43) Ellis, R. Journal of Physical Chemistry B 2014, 118, 315-322.

515 (44) Coniglio, A.; Stanley, H. E.; Klein, W. Physical Review B 1982, 25, 6805-6821. 


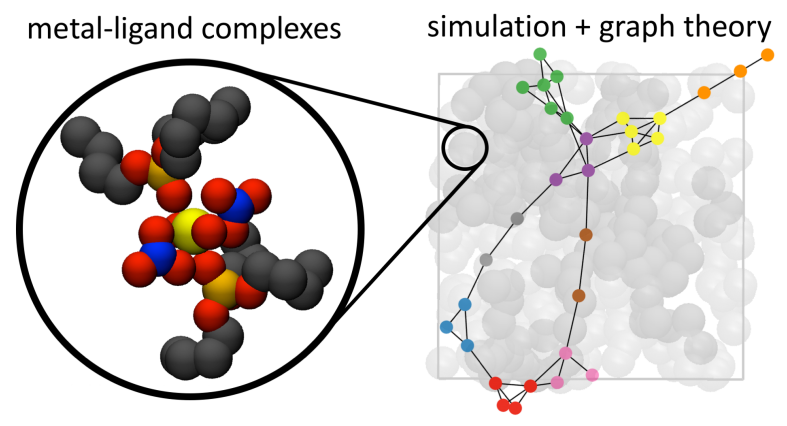

Figure 7: TOC image. 